\title{
Evaluating Influential Factors on the Duration of Vehicle Fire Incidents Using Grey Relational Analysis
}

\author{
Wenhui Zhang, Jing Dai, Yongmin Su, Qiqi Miao, Feng Guan and Zhiwei Gong \\ Department of Transportation, Northeast Forestry University, Harbin, Heilongjiang Province, 150040, China
}

\begin{abstract}
The duration of vehicle fire incidents has been closely associated with incidents loss. Understanding the influential priority of factors is significant to take targeted countermeasures for the managements. Based on the database from WSDOT (Washington Department of Transportation) in USA, we analyze the probability distribution of the vehicle fire accidents' duration. Then we classify the influential factors into the first-grade factors including three categories: time, incident type, operation and the second-grade factors including eight categories: quarter, week and day time, etc. Then GRA (grey relational analysis) model is applied to calculate grey relational grades of the influential factors. The results show that the most important factor of the first-grade factors is incident type, vehicles involved and agencies involved are the major factors among the second-grade factors.
\end{abstract}

Key words: Incident duration, vehicle fire, influential factors, grey relational analysis.

\section{Introduction}

Vehicle fire incidents lead to a large amount or casualty and property loss, longer traffic congestion, emission and other potential danger. Therefore, an effective approach [1] to avoid vehicle fire incidents is a primary task for TIMS (traffic incident management system). However, the duration of vehicle fire accident is another importance which requires to estimate the influential factors of vehicle fire incidents duration [2]. Total vehicle fire incident duration which is similar to vehicle incidents duration [3] can be divided into four time intervals. This paper, we only consider the total duration.

Most of the previous studies focused on estimation of traffic incident duration. A retrospective population-based case-control study was performed to determine the association between vehicle fires [4]. Four full-scale car fire experiments were carried out to determine the burning behavior of 4-door sedan passenger cars [5]. A novel approach for accident duration prediction is proposed, in which the leaves of

\footnotetext{
Corresponding author: Wenhui Zhang, professor; research field: transportation safety. E-mail addresses: zhangwenhui@nefu.edu.cn.
}

the tree model are HBDMs (hazard-based duration models) instead of linear regression models [6]. A competing risk mixture hazard-based model is proposed to analyze the effect of various factors on traffic incident duration and predict the duration sequentially [7].

However, limited literature concentrated on evaluating influential factors on the duration of vehicle fire incidents. Exploring features of these fire accidents was significant for both transit companies and insurance companies. And a great number of studies have proven that GAR (grey relational analysis) $[8,9]$ is effective for evaluating relationships between influential factors and systems. To this extent, we will explore the influential priority of factors by use of GRA. So, this paper is constructed as follows. In Section 2, the data source and descriptions are summarized. GRA which is used to identify the main influential factors are briefly described in Section 3. And Section 4 presents the results and discussions. At last, the conclusions and future studies are provided.

\section{Date Source and Description}

The duration data of vehicle fire incidents used in 
this study were collected from the WITS (Washington Incident Tracking System) database maintained by the WSDOT (Washington Department of Transportation). There are a total of 1,018 vehicle fire incidents cases recorded from January 1 in 2002 to December 31 in 2013. The dataset included the time, incident type, operation, agencies involved, vehicles involved, detailed description, ect. [10]. Table 1 illustrated the primary statistics of the vehicle fire incident duration data consisting of response time and clearance time.

From the table, the mean values of incident response time, clearance time and duration are, respectively, 10.08 minutes, 63.42 minutes and 73.49 minutes.

In order to observe the probability distribution of vehicle fire incidents duration including response time and clearance time, we applied the corresponding frequency distribution histogram, as shown in Fig. 1. The horizontal axis of histogram showed data packets and the vertical axis showed frequency, and probability density function could express this distribution. It could be found that the longer duration corresponds with the smaller proportion, as well as response time and clearance time, and log-logistic

Table 1 Statistics summaries for vehicle fire incident duration data.

\begin{tabular}{lllllll}
\hline Statistic & Sample size & Max $(\mathrm{min})$ & Min $(\mathrm{min})$ & Mean $(\mathrm{min})$ & Std. dev. & Skewness \\
\hline Response time & 1,018 & 90 & 0 & 10.08 & 9.96 & 3.33 \\
Clearance time & 1,018 & 1,030 & 1 & 63.42 & 90.64 & 5.24 \\
Duration & 1,018 & 1,075 & 1 & 73.49 & 94.99 & 4.91 \\
\hline
\end{tabular}

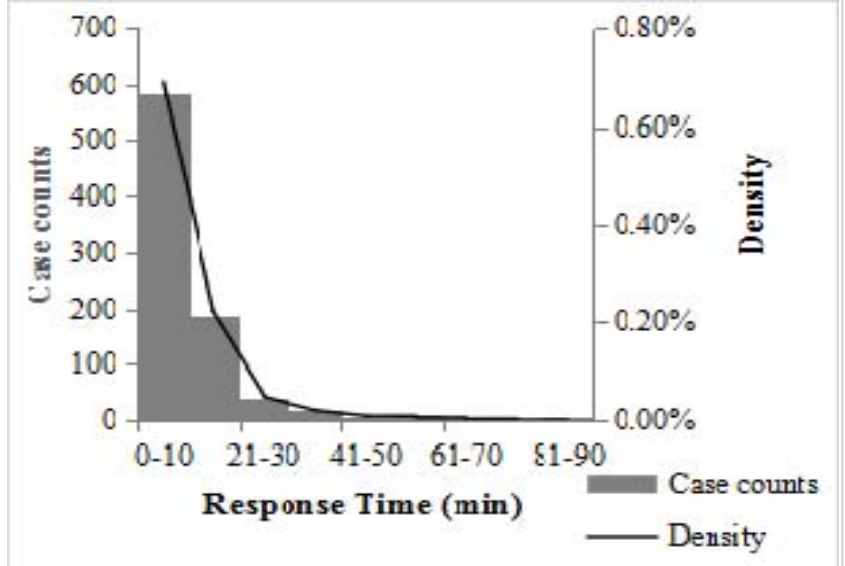

(a)

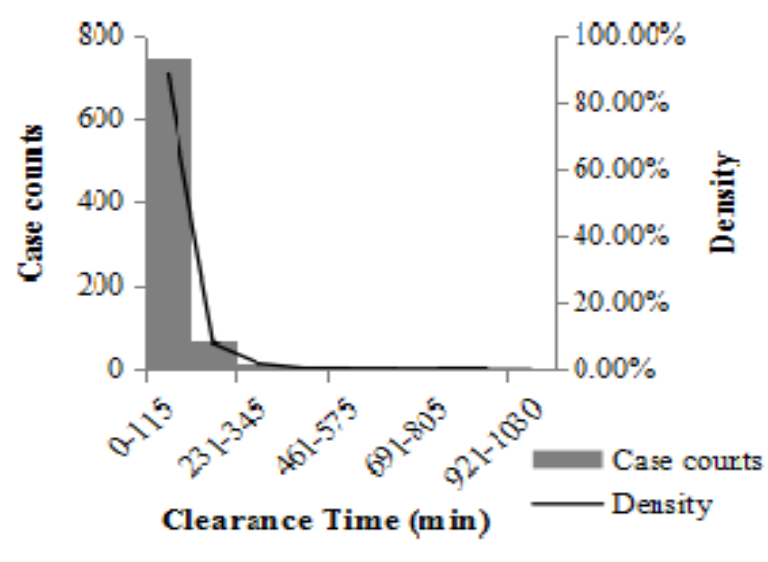

(b)

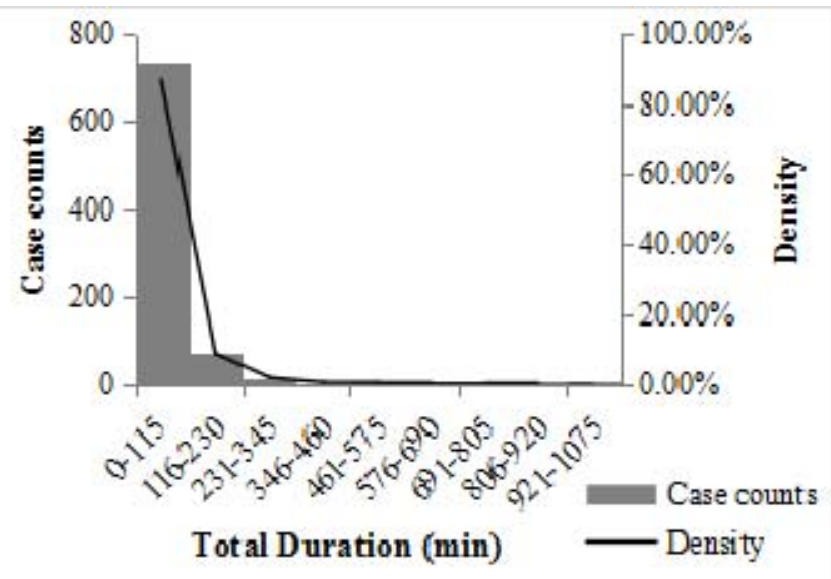

(c)

Fig. 1 Fitting distribution of vehicle fire incident duration: (a) response time; (b) clearance time; (c) total duration. 
distribution fitted the best.

Box plot was employed to reveal the relationships between each factor and duration of vehicle fire incidents, as shown in Fig. 2. It could be concluded that the vehicle fire incidents occurring in Quarter 4, Sunday or early morning of time category tended to cause higher duration. If an incident invovled one vehicle occurred, other fire type or five lanes blocking of incident type category, the duration tended to be higher. The duration was higher if the incident spot took traffic control measures or WSP and WSDOT agencies involved of operation category. However, the influential priority of eight factors could not be found from Fig. 2.
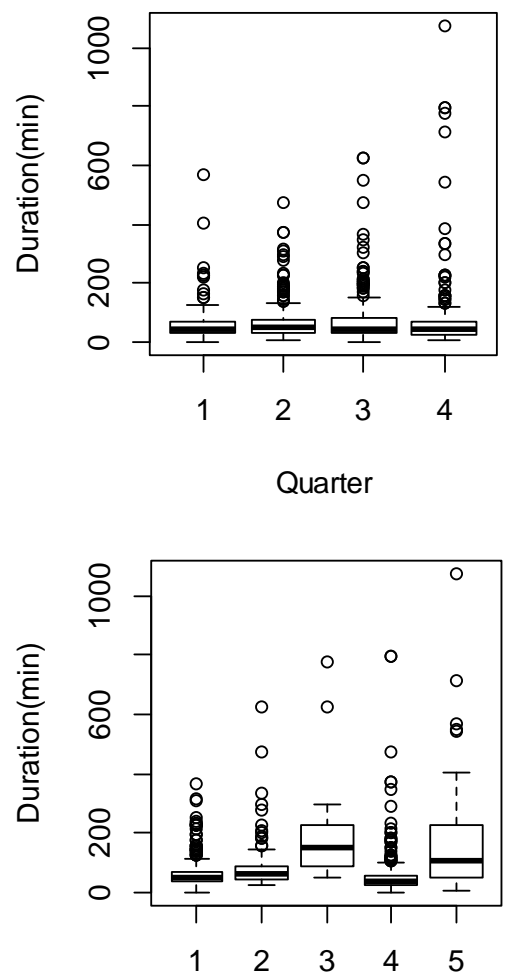

Lanes Blocking

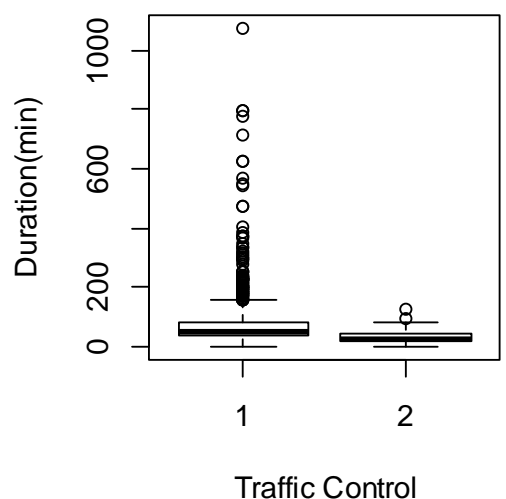

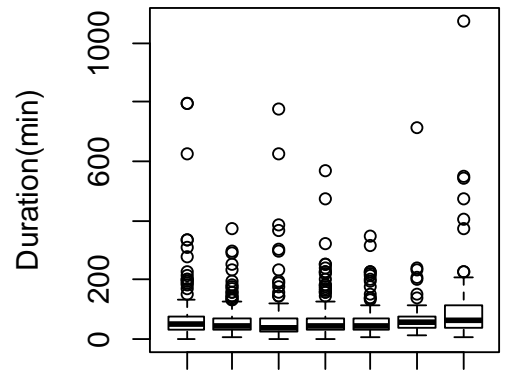

$\begin{array}{lllllll}1 & 2 & 3 & 4 & 5 & 6 & 7\end{array}$

Week

(a)

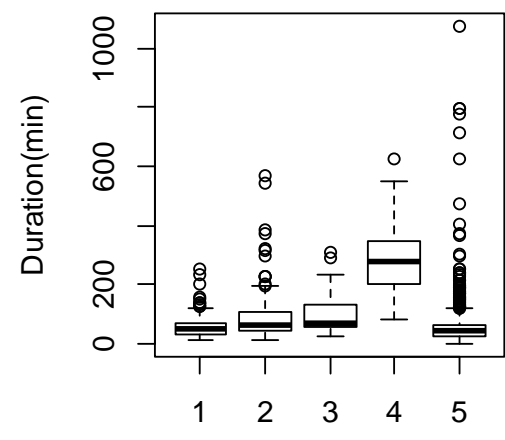

Fire Type

(b)

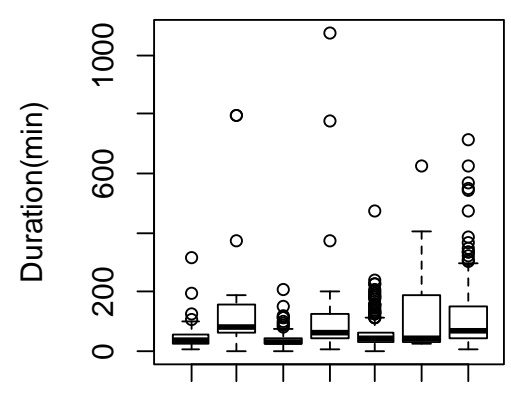

$\begin{array}{lllllll}1 & 2 & 3 & 4 & 5 & 6 & 7\end{array}$

Agencies Involved

(c)

Fig. 2 Boxplot of factors and Duration: (a) time; (b) incident type; (c) operation. 


\section{Methodology}

\subsection{Grey Relational Generating}

If the number of alternatives is $m$ and the number of attributes is $n$, the alternatives can be expressed as $X i$ $=\left\{x_{i}(1), x_{i}(2), \ldots, x_{i}(n)\right\}$. And according to the following three equations which are used for the normalization respectively, the term $X_{i}$ can be translated into the comparability sequences $X_{i}^{\prime}=\left\{x_{i}^{\prime}(1), x_{i}^{\prime}(2), \cdots, x_{i}^{\prime}(n)\right\}$.

If the expectation is the-larger-the-better, it can be calculated the following equation:

$$
\begin{gathered}
X_{i}^{\prime}=\frac{x_{i}(k)-\min x_{i}(k)}{\max x_{i}(k)-\min x_{i}(k)} \text {, for } i=1,2, \cdots, m ; k=1,2, \cdots n \text { (1) } \\
\text { If the } \gamma_{i}=\frac{1}{N} \sum_{k=1}^{N} \xi_{i}(k) \quad \text { expectation is }
\end{gathered}
$$
the-smaller-the-better, it can be calculated the following equation:

$$
X_{i}^{\prime}=\frac{\max x_{i}(k)-x_{i}(k)}{\max x_{i}(k)-\min x_{i}(k)}, \text { for } i=1,2, \cdots, m ; k=1,2, \cdots n(2)
$$

\subsection{Reference Sequence Definition}

After the grey relational generating procedure using Eqs. (1), (2) or (3), all performance values will be scaled into $[0,1]$. If the evaluation index of an alternative by grey relational generating procedure tends to 1 than others, that means the evaluation index is one of the best attributes. Therefore, for convenience, we defines the reference sequence $X_{0}$ as $\left\{x_{0}(1), x_{0}(2), \ldots, x_{0}(n)\right\}=(1,1, \ldots, 1)$.

\subsection{Grey Relational Coefficient Calculation}

For a reference sequence has several comparability sequences and based on the normalized data, the grey relational coefficient can be calculated by the following formula:

$$
\xi_{i}(k)=\frac{\Delta \min +\rho \Delta \max }{\Delta_{o i}(k)+\rho \Delta \max }, \text { for } i=1,2, \cdots, m ; k=1,2, \cdots, n(4)
$$

where, $\rho$ is the distinguish coefficient which is usually between 0 and 1 , and generally value of 0.5 .

\subsection{Grey Relational Grade Calculation}

It is necessary to focus on a value of relational coefficient, which is its average. And the grey relational grade can be calculated by the following equation:

$$
\gamma_{i}=\frac{1}{N} \sum_{k=1}^{N} \xi_{i}(k)
$$

where, $\gamma_{i}$ is the grey relational grade between $X_{0}$ and $X_{i}^{\prime}, N$ is the number of performance characteristics.

\section{Results and Discussion}

Grey relational analysis was performed to evaluate the influential priority of the various factors on the vehicle fire incident duration. Prior to calculation, all the data were normalized using Eq. (1). Then the grey relational coefficients were computed using Eq. (4). The grey relational grades were calculated using Eq. (5).

On the analysis of the total fire duration before, we first set out to describe the two aspects consisting of response time and clearance time.

In order to observe the influential priority of eight factors on the response time in-depth, the data was divided into some small intervals that each is $15 \mathrm{~min}$ in which the grey relational grades were calculated respectively. Table 2 showed the grey relational grades of all the influential factors of response time.

According to Eqs. (1)-(5), we can calculate GRG (grey relational grades).

From the table, it showed that the first three influential factors of response time was traffic control $>$ agencies involved $>$ day time. So, the three factors made largest contribution to response time. And also it demonstrated that the grey relational grades decreased in turn from Section 1 to Section 5, the reason was that Section 1 which is 0 to 15 minutes accounts for about $70 \%$, and the later was less. Therefore, Section 1 was more convincing. But its grey relational grades of all the factors were consistent with the influential priority of response time. 
Table 2 Grey relational grades of factors to response time.

\begin{tabular}{llllllll}
\hline \multirow{2}{*}{ Factors } & \multirow{2}{*}{ Response time } & \multicolumn{5}{c}{ Response time interval } \\
\cline { 5 - 8 } & & & 1 & 2 & 3 & 4 & 5 \\
\multirow{3}{*}{ Time } & Quarter & 0.7506 & 0.8000 & 0.8500 & 0.8489 & 0.7144 & 0.5397 \\
& Week & 0.6901 & 0.7929 & 0.8133 & 0.7702 & 0.5931 & 0.4811 \\
& Day time & 0.7587 & 0.8552 & 0.8116 & 0.812 & 0.671 & 0.6435 \\
\hline \multirow{3}{*}{ Incident type } & Lanes blocking & 0.7425 & 0.7828 & 0.7921 & 0.7097 & 0.7226 & 0.7053 \\
& Fire type & 0.7314 & 0.7998 & 0.8537 & 0.8368 & 0.6734 & 0.4933 \\
& Vehicle involved & 0.6930 & 0.7987 & 0.8377 & 0.6908 & 0.5115 & 0.6261 \\
\hline \multirow{2}{*}{ Operation } & Traffic control & 0.8453 & 0.9025 & 0.8945 & 0.8109 & 0.8311 & 0.7874 \\
& Agencies involved & 0.7642 & 0.9015 & 0.8670 & 0.8009 & 0.6716 & 0.5800 \\
\hline
\end{tabular}

Table 3 Grey relational grades of factors to clearance time.

\begin{tabular}{llllllll}
\hline \multirow{2}{*}{ Factors } & \multirow{2}{*}{ Clearance time } & \multicolumn{5}{c}{ Clearance time interval } \\
\cline { 4 - 8 } & & 1 & 2 & 3 & 4 & 5 \\
\hline \multirow{3}{*}{ Time } & Quarter & 0.6867 & 0.7911 & 0.7889 & 0.6616 & 0.5608 & 0.6310 \\
& Week & 0.6489 & 0.7666 & 0.7703 & 0.6495 & 0.5675 & 0.4908 \\
& Day time & 0.6509 & 0.7905 & 0.8245 & 0.6095 & 0.6187 & 0.4112 \\
\hline \multirow{3}{*}{ Incident type } & Lanes blocking & 0.7282 & 0.7370 & 0.7834 & 0.7198 & 0.6858 & 0.7150 \\
& Fire type & 0.7763 & 0.7726 & 0.8515 & 0.7230 & 0.6926 & 0.8418 \\
& Vehicle involved & 0.7862 & 0.7956 & 0.8776 & 0.8062 & 0.8713 & 0.5801 \\
\hline \multirow{2}{*}{ Operation } & Traffic control & 0.7491 & 0.7649 & 0.8231 & 0.7059 & 0.7100 & 0.7418 \\
& Agencies involved & 0.8508 & 0.7788 & 0.9016 & 0.8040 & 0.9278 & 0.8418 \\
\hline
\end{tabular}

Table 4 Grey relational grades of factors to vehicle fire incident duration.

\begin{tabular}{llllllll}
\hline \multirow{2}{*}{ Factors } & \multirow{2}{*}{ Duration } & \multicolumn{5}{c}{ Duration interval } \\
\cline { 4 - 8 } & & & 1 & 2 & 3 & 4 & 5 \\
\multirow{3}{*}{ Time } & Quarter & 0.6612 & 0.8353 & 0.8032 & 0.5608 & 0.5826 & 0.5239 \\
& Week & 0.6153 & 0.6975 & 0.7178 & 0.5132 & 0.6180 & 0.5301 \\
& Day time & 0.6531 & 0.8353 & 0.7557 & 0.5938 & 0.6475 & 0.4330 \\
\hline \multirow{3}{*}{ Incident type } & Lanes blocking & 0.6726 & 0.4330 & 0.7092 & 0.6953 & 0.7214 & 0.8043 \\
& Fire type & 0.7861 & 0.8077 & 0.8343 & 0.7721 & 0.6963 & 0.8202 \\
& Vehicle involved & 0.8772 & 0.8939 & 0.9062 & 0.9274 & 0.8381 & 0.8202 \\
\hline \multirow{2}{*}{ Operation } & Traffic control & 0.7046 & 0.8058 & 0.7597 & 0.5713 & 0.6259 & 0.7605 \\
& Agencies involved & 0.8259 & 0.8622 & 0.8919 & 0.7118 & 0.8583 & 0.8053 \\
\hline
\end{tabular}

And the clearance time data was also divided into some small intervals that each is 1,115 min in which the grey relational grades were calculated respectively. Table 3 showed the grey relational grades of all the influential factors of clearance time.

It is obviously that the largest grey relational grade is agencies involved, and ranking influential factors are agencies involved $>$ vehicle involved $>$ fire type. However, in Section 1 and 2, the three factors of time category had significant influence on the clearance time and all the factors of incident type and operation categories in which the grey relational grades had no significant difference had significant influence on it .

Similarly, in order to distinguish the influential priority of eight factors on the duration in-depth, the data was divided into some small intervals that each is 115 minutes in which the grey relational grades were calculated respectively. Table 4 showed the grey relational grades of all the influential factors of duration.

It indicated that the influential priority of the first-grade factors on vehicle fire incidents duration 
was incident type $>$ operation $>$ time, the second-grade factors were vehicle involved $>$ agencies, involved $>$ fire type $>$ traffic control $>$ lanes blocking $>$ quarter $>$ day time $>$ week. The results show that the most important factor of the first-grade factors is incident type, vehicles involved and agencies involved are the major factors among the second-grade factors. In addition, in Sections 1 to 5, the three factors had significant influence on the duration, respectively.

We can come to the conclusions that the order of influential factors of response time is different from clearance time and total duration. Nevertheless, clearance time and total duration have the same first three influential factors.

\section{Conclusions}

The vehicle incidents duration that resulted in fire has direct relationship with body damage and property loss. Based on 1,018 records from WSDOT, this study took the eight variables as influence factors and applied GRA to explore the influence priority of factors. According to the results of study, we can draw the conclusion that vehicle involved, agencies involved and incident type have extremely impact on vehicle fire incidents duration. These findings may help the related management agencies try to take targeted countermeasures to reduce the body damage and property loss caused by fire incident.

\section{Acknowledgments}

This work is financially supported by the Fundamental Research Funds for the Central
Universities (2572015CB13) and General Project of Humanities and Social Sciences Research of Ministry of Education (17YJCZH250).

\section{References}

[1] Wang, Y., and Chu, J. 2014. "Diagnosis Information Extraction of Misfire Fault of Vehicle Electronically Controlled Engine Based on Daubechies Wavelet." Forestry Engineering 30 (02): 138-42, 166.

[2] Yan, G., and Rawat, D. B. 2016. "Vehicle-to-Vehicle Connectivity Analysis for Vehicular Ad-Hoc Networks.” Ad Hoc Networks 74: 77-86.

[3] Zhan, C., Gan, A., and Hadi, M. 2011. "Prediction of Lane Clearance Time of Freeway Incidents Using the M5P Tree Algorithm.” Intelligent Transportation Systems IEEE Transactions 12 (4): 1549-57.

[4] Bunn, T. L., Slavova, S., and Robertson, M. 2012. "Crash and Burn? Vehicle, Collision, and Driver Factors That Influence Motor Vehicle Collision Fires.”Accident; Analysis and Prevention 47 (47): 140-5.

[5] Zhang, W., and Ma, J. 2017. "The Identification of Overtaking Risk Factors on Two Lane Highways.” Forestry Engineering 33 (03): 89-93.

[6] Lin, L., Wang, Q., and Sadek, A. W. 2016. “A Combined M5P Tree and Hazard-Based Duration Model for Predicting Urban Freeway Traffic Accident Durations.” Accident Analysis \& Prevention 91: 114-26.

[7] Hu, M., and Yang, B. 2014. "Parameters Study on Head Finite Element Model during Vehicle Collision Accident.” Forestry Engineering 30 (06): 89-93.

[8] Benavides, R., and Escobar, R. 2017. "Resurgence of Response Duration in Human Participants.” Behavioural Processes 142: 106.

[9] Wagner, V., Kallus, K. W., and Foehl, U. 2017. "Dimensions of Vehicle Sounds Perception.” Applied Ergonomics 64: 41.

[10] Chimba, D., and Kutela, B. 2014. "Scanning Secondary Derived Crashes from Disabled and Abandoned Vehicle Incidents on Uninterrupted Flow Highways.” Journal of Safety Research 50 (5): 109-16. 